\title{
High Dose Chemotherapy
}

National Cancer Institute

\section{Source}

National Cancer Institute. High Dose Chemotherapy. NCI Thesaurus. Code C15807.

A cancer treatment having a treatment dose that is higher than the standard dosage levels recommended on the product labeling for that chemotherapeutic agent. This dose is concurrently toxic and destroys the normal bone marrow stem cells. 